\title{
Reduced Order Modeling of Turbulent Flows Using Statistical Coarse-graining
}

\author{
Eric Parish* and Karthik Duraisamy ${ }^{\dagger}$ \\ Department of Aerospace Engineering, University of Michigan, Ann Arbor, MI 48109.
}

\begin{abstract}
Traditional sub-grid models for Large Eddy Simulations (LES) of turbulence rely on theoretical and physical arguments and are known to be inadequate in many applications. This work investigates the use of the Mori-Zwanzig formalism, a concept that originates from non-equilibrium statistical mechanics, to develop sub-grid models for LES. The mechanics of the generalized Langevin equation (GLE) are considered and a methodology for directly solving the orthogonal dynamics equation is presented. This process is shown to provide insight into the form of the GLE and the effects of coarse-graining. This knowledge is used to derive a class of sub-grid models that are capable of consistently representing non-local memory effects. The models are applied to the viscous Burgers equation and homogeneous isotropic turbulence. The models are shown to be in good agreement with high resolution simulations and outperform Smagorinsky-type models.
\end{abstract}

\section{Introduction}

In Large Eddy Simulations of turbulent flows, the effect of the unresolved (or microscopic) scales on the resolved (or macroscopic) scales is expressed through a sub-grid model. The development of coarse-grained models for systems that display a continuous cascade of scales is challenging. The continuous interaction between the resolved and unresolved scales leads to non-local effects that are challenging to understand and model. Traditional sub-grid models are based on theoretical arguments and physical observations, such as homogeneity and scale invariance, and are known to be inadequate in many problems of engineering interest.

The Mori-Zwanzig (M-Z) formalism ${ }^{1,2}$ provides a formal setting for the development of coarse-grained models from the viewpoint of statistical mechanics. The M-Z formalism casts a dynamical system into an equivalent lower dimensional system governed by the generalized Langevin equation (GLE). While the GLE is an exact representation of the dynamics, it involves a convolution integral representing non-local effects. Thus, expressing unresolved content as a function of the resolved scales requires the inclusion of memory effects. The Mori-Zwanzig formalism alone does not lead to a reduction in complexity as it requires the solution of the orthogonal dynamics equation to compute the convolution term.

In recent years, however, the Mori-Zwanzig formalism has gained attention within the setting of model order reduction. The optimal prediction framework developed by the Chorin group ${ }^{3-7}$ uses the GLE to obtain equations that describe the evolution of the macroscopic scales conditioned on the knowledge of the microscopic scales. The framework removes the dependence of the GLE on the unresolved terms and begins to address how the memory can be approximated without solving the orthogonal dynamics equation.

Approximating the memory convolution integral requires insight into the orthogonal dynamics. Givon et al. ${ }^{8}$ prove that classical solutions exist to the orthogonal dynamics equation for a simple set of projection operators and show the existence of weak solutions in the general case. Chorin et al. ${ }^{4}$ make use of the fact that the Hermite polynomials are orthogonal with respect to the conditional expectation inner product and develop a set of Volterra integral equations that can be used to approximate the memory integrand. This finite-rank projection is shown to provide a reasonably accurate representation of the memory kernel for a system of two oscillators with a non-linear coupling, but the process is intractable for high-dimensional problems unless a low-dimensional basis is used. Bernstein ${ }^{9}$ uses a first order basis

\footnotetext{
${ }^{*}$ PhD Candidate, Department of Aerospace Engineering, University of Michigan, Ann Arbor

${ }^{\dagger}$ Assistant Professor, Department of Aerospace Engineering, University of Michigan, Ann Arbor
} 
to attempt to gain information about the memory kernel for Burgers equation. The simplicity of the basis functions, however, limit the insight gained from this process. In addition to dimensional considerations, the finite rank projection has an additional deficiency in that the form of the integrand may be incorrect. This limits the ability to test theories such as a finite support of the memory integrand.

Despite the absence of information about the form of the memory, various surrogate models exist. The crudest and perhaps most common approximation is the t-model, so named because time appears explicitly in the model form. The t-model is derived by assuming the memory is infinitely long and leads to the memory being approximated by a Markovian term. The t-model has been applied to a variety of problems, including the Burgers ${ }^{9}$ and Euler equations. Chandy and Frankel ${ }^{10}$ used the t-model for coarse-grained simulations of homogeneous isotropic turbulence and the Taylor-Green vortex. The model was found to perform well for low Reynolds number cases, but significant discrepancies were seen for higher Reynolds numbers. Stinis ${ }^{11}$ introduced a set of renormalized models based on expansions of the memory integrand. These models were applied to Burgers equation and were found to be in good agreement with the full order model.

A class of more sophisticated approximations to the memory term was derived by Stinis. ${ }^{12,13}$ These approximations involve developing an infinite set of Markovian differential equations that describe the evolution of the memory integral. The process uses discrete integration schemes to cast the additional equations in a form that is independent of the orthogonal dynamics. The models were applied to Burgers equation and the 3D Euler equations. In the numerical experiments it was found that a finite memory length was required for stability.

The objective of the current work is to demonstrate the predictive capabilities of the Mori-Zwanzig based models and to provide a deeper investigation into the form of the memory kernel. A methodology for constructing an approximate analytic memory integrand is introduced and the models will be applied to the viscous Burgers equation and the incompressible Navier-Stokes equations.

\section{The Mori-Zwanzig Formalism}

A discussion of the Mori-Zwanzig formalism and the optimal prediction framework developed by Chorin and collaborators is provided in this section. Detailed discussions can be found in Refs. 4, 5. Consider the semi-discrete non-linear ODE

$$
\frac{d \phi}{d t}=R(\phi),
$$

where $\phi=\{\hat{\phi}, \tilde{\phi}\}$, with $\hat{\phi} \in \mathbb{R}^{N}$ being the resolved modes, and $\tilde{\phi} \in \mathbb{R}^{M}$ being the unresolved modes. The initial condition is $\phi(0)=\phi_{0}$. The non-linear ODE can be posed as a linear partial differential equation by casting it in the Liouville form,

$$
\frac{\partial}{\partial t} u\left(\phi_{0}, t\right)=\mathcal{L} u\left(\phi_{0}, t\right)
$$

with $u\left(\phi_{0}, 0\right)=g\left(\phi\left(\phi_{0}, 0\right)\right)$. The Liouville operator is defined as

$$
\mathcal{L}=\sum_{k=1}^{N+M} R_{k}\left(\phi_{0}\right) \frac{\partial}{\partial \phi_{0 k}}
$$

where $\phi_{0 k}=\phi_{k}(0)$. The solution to Eq. 2 can be verified to be

$$
u\left(\phi_{0}, t\right)=g\left(\phi\left(\phi_{0}, t\right)\right) .
$$

Using the semigroup notation, Eq. 2 can be expressed as

$$
\frac{\partial}{\partial t} g\left(e^{t \mathcal{L}} \phi_{0}\right)=g\left(\mathcal{L} e^{t \mathcal{L}} \phi_{0}\right)
$$

Consider the initial conditions to be random variables drawn from some probability distribution $\mathrm{P}\left(\phi_{0}\right)$. Given this distribution, the expected value of a function $g\left(\phi_{0}\right)$ is given by

$$
\mathrm{E}\left[g\left(\phi_{0}\right)\right]=\int_{\Gamma} g\left(\phi_{0}\right) \rho\left(\phi_{0}\right) d \phi_{0}
$$

where $\rho$ is the probability density. Assume $\phi \in \Gamma$, where $\Gamma$ is an $L^{2}$ Hilbert space endowed with an inner product $(f, g)=\mathrm{E}[f g]$. Consider now the coarse-grained simulation of Eq. 1, where the variables in $\hat{\phi}$ are resolved and the 
variables in $\tilde{\phi}$ are unresolved. By taking $g\left(\phi_{0}\right)=\phi_{0 j}$, an equation for the trajectory of a resolved variable can be written as

$$
\frac{\partial}{\partial t} e^{t \mathcal{L}} \phi_{0 j}=e^{t \mathcal{L}} \mathcal{L} \phi_{0 j}
$$

The term on the right hand side is a function of both the resolved and unresolved variables. To proceed, define the space of the resolved variables by $\hat{L}^{2}$ and the unresolved variables by $\tilde{L}^{2}$. Further, define $P: L^{2} \rightarrow \hat{L}^{2}$, as well as $Q=I-\mathcal{P}$. An example of a possible projection operator would be, for a function $f\left(\hat{\phi}_{0}, \tilde{\phi}_{0}\right), \mathcal{P} f\left(\hat{\phi}_{0}, \tilde{\phi}_{0}\right)=f\left(\hat{\phi}_{0}, 0\right)$. Using the identity $I=\mathcal{P}+Q$, the right hand side of Eq. 5 can be split into a component that depends on the resolved variables and one that depends on the unresolved variables,

$$
\frac{\partial}{\partial t} e^{t \mathcal{L}} \phi_{0 j}=e^{t \mathcal{L}} \mathcal{P} \mathcal{L} \phi_{0 j}+e^{t \mathcal{L}} Q \mathcal{L} \phi_{0 j}
$$

At this point the Duhamel formula is utilized,

$$
e^{t \mathcal{L}}=e^{t Q \mathcal{L}}+\int_{0}^{t} e^{(t-s) \mathcal{L}} \mathcal{P} \mathcal{L} e^{s Q \mathcal{L}} d s .
$$

Inserting the Duhamel formula into Eq. 6, the generalized Langevin equation is obtained,

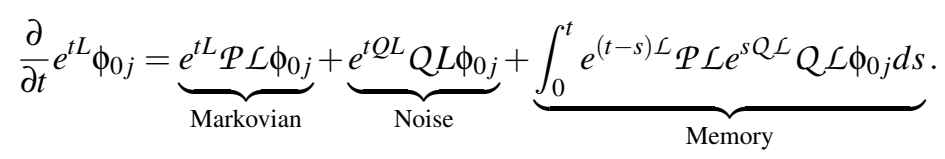

Eq. 7 is the Mori-Zwanzig identity. The system described in Eq. 7 is exact and is an alternative way of expressing the original system. Eq. 7 makes a profound statement: coarse-graining leads to memory effects. For notational purposes, define

$$
F_{j}\left(\phi_{0}, t\right)=e^{t Q \mathcal{L}} Q \mathcal{L} \phi_{0 j} \quad K_{j}\left(\phi_{0}, t\right)=\mathcal{P} \mathcal{L} F_{j}\left(\phi_{0}, t\right) .
$$

By definition, $F_{j}\left(\phi_{0}, t\right)$ satisfies

$$
\frac{\partial}{\partial t} F_{j}\left(\phi_{0}, t\right)=Q \mathcal{L} F_{j}\left(\phi_{0}, t\right)
$$

where $F_{j}\left(\phi_{0}, 0\right)=Q \mathcal{L} \phi_{0 j}$. Eq. 9 is referred to as the orthogonal dynamics equation. It can be shown that solutions to the orthogonal dynamics equation live in the null space of $\mathcal{P}$ for all time, meaning $\mathcal{P} F_{j}\left(\phi_{0}, t\right)=0$. Using the notation in Eq. 8, Eq. 7 can be written as

$$
\frac{\partial}{\partial t} \phi_{j}\left(\phi_{0}, t\right)=R_{j}\left(\hat{\phi}\left(\phi_{0}, t\right)\right)+F_{j}\left(\phi_{0}, t\right)+\int_{0}^{t} K_{j}\left(\hat{\phi}\left(\phi_{0}, t-s\right), s\right) d s .
$$

A simplification comes from projecting Eq. 10 to eliminate the dependence on the noise term,

$$
\frac{\partial}{\partial t} \mathcal{P} \phi_{j}\left(\phi_{0}, t\right)=\mathcal{P} R_{j}\left(\hat{\phi}\left(\phi_{0}, t\right)\right)+\mathcal{P} \int_{0}^{t} K_{j}\left(\hat{\phi}\left(\phi_{0}, t-s\right), s\right) d s
$$

Eq. 11 provides a set of equations for $\mathcal{P} \phi\left(\phi_{0}, t\right)$, the best possible approximation for $\hat{\phi}$ on $\hat{L}^{2}$ given knowledge about the initial density of $\tilde{\phi}$. Note that the final projection does not necessarily imply a reduction in computational complexity. Although Eq. 11 has no dependence on the noise term, one is left with the challenge of projecting the potentially non-linear Markovian term. For general non-linear functions, the projection operator does not commute $(\mathrm{E}[f(x)] \neq$ $f(\mathrm{E}[x]))$.

Various projection operators that satisfy $\mathcal{P}: L^{2} \rightarrow \hat{L}^{2}$ exist. This work utilizes the expectation projection. Let $f \in L^{2}$. The projection of $f$ onto $\hat{L}^{2}$ is given by

$$
(\mathcal{P} f)\left(\hat{\phi}_{0}\right)=E\left[f \mid \hat{\phi}_{0}\right]=\frac{\int f\left(\phi_{0}\right) \rho\left(\phi_{0}\right) d \tilde{\phi}_{0}}{\int \rho\left(\phi_{0}\right) d \tilde{\phi}_{0}} .
$$

In this work the density is taken to consist of independent Gaussian distributions centered about zero in the zerovariance limit as in Ref. 9. Physically, this corresponds to the case where the initial conditions of the unresolved modes are known to initially be zero. The commutation of the projection operator is valid under this simplification as the unresolved degrees of freedom in the initial conditions are eliminated. 


\section{The Orthogonal Dynamics Equation and the Memory Kernel}

The challenge of the M-Z procedure for model order reduction is to construct an accurate, computationally tractable approximation to the memory integral. The construction of such an approximation requires an understanding of the form of the integrand and the underlying mechanics of the memory kernel. In its primitive form, the orthogonal dynamics equation (Eq. 9) is a partial differential equation that has dimension $N+M$ (with $N$ being the number of resolved variables and $M$ being the number of unresovled variables.) Pursuing solutions to the orthogonal dynamics in this form is not tractable. Recall, however, that the generalized Langevin equation itself is a PDE with dimension $N+M$, but its solution may be obtained by solving a set of ordinary differential equations. As such, we hypothesize that a set of ordinary differential equations exist that satisfy the orthogonal dynamics equation.

Define an auxiliary semi-discrete system

$$
\frac{d}{d t} \phi_{j}^{Q}=R_{j}^{Q}\left(\phi^{Q}\left(\phi_{0}, t\right)\right)
$$

with $\phi^{Q} \in \tilde{L}^{2}, \phi_{j}^{Q}(0)=\phi_{0 j}$. Additionally, define an auxiliary Liouville operator,

$$
\mathcal{L}^{Q}=\sum_{i=1}^{N+M} R_{i}^{Q}\left(\phi_{0}, 0\right) \frac{\partial}{\partial \phi_{0 i}}
$$

The auxiliary system can be cast into Liouville form

$$
\frac{\partial}{\partial t} F\left(\phi_{0}, t\right)=\mathcal{L}^{Q} F\left(\phi_{0 j}, t\right)=\sum_{i=1}^{N+M} R_{i}^{Q}\left(\phi_{0}, 0\right) \frac{\partial}{\partial \phi_{0 i}} F\left(\phi_{0}, t\right),
$$

with $F\left(\phi_{0}, 0\right)=b\left(\phi_{0}, 0\right)$. It can be verified that the solution to Eq. 13 is given by

$$
F\left(\phi_{0}, t\right)=b\left(\phi^{Q}\left(\phi_{0}, t\right)\right)
$$

By definition, the orthogonal dynamics equation (Eq. 9) satisfies

$$
\frac{\partial}{\partial t} F\left(\phi_{0}, t\right)=Q \mathcal{L} F=Q\left[\sum_{i=1}^{N+M} R_{i}\left(\phi_{0}, 0\right) \frac{\partial}{\partial \phi_{0 i}} F\left(\phi_{0}, t\right)\right] .
$$

Therefore it is seen that the orthogonal dynamics equation can be solved by evolving a set of ordinary differential equations if

$$
\sum_{i=1}^{N+M} R_{i}^{Q}\left(\phi_{0}, 0\right) \frac{\partial}{\partial \phi_{0 i}} F\left(\phi_{0}, t\right)=Q\left[\sum_{i=1}^{N+M} R_{i}\left(\phi_{0}, 0\right) \frac{\partial}{\partial \phi_{0 i}} F\left(\phi_{0}, t\right)\right] .
$$

Given the proper choice of $R_{i}^{Q}$, a set of ordinary differential equations can be derived that satisfy the orthogonal dynamics. The form of this set of ordinary differential equations is dependent on the projection operator used, the initial conditions, etc. As an example, for some systems the property can be obtained by setting $R_{i}^{Q}\left(\phi_{0}\right)=\mathcal{L} \phi_{0}-\mathcal{P} \mathcal{L} \phi_{0}$.

Assuming the existence of the auxiliary set of ordinary differential equations, the memory can be written without approximation to be

$$
\int_{0}^{t} e^{(t-s) \mathcal{L}} \mathcal{P} \mathcal{L} e^{s Q \mathcal{L}} Q \mathcal{L} \phi_{0 j} d s=\int_{0}^{t} \mathcal{P} \mathcal{L} Q \mathcal{L} \phi_{j}^{Q}(\phi(t-s), s) d s
$$

Although Eq. 14 is exact and obtaining solutions to the orthogonal dynamics may be tractable numerically, challenges are still present. In particular, note that the memory integrand involves evaluation of $\phi^{Q}(\phi(t-s), s)$. The practical consequence is that, when solved numerically, the dependence of the orthogonal dynamics on the initial conditions is not known analytically.

To gain insight into the memory term, a simplification is considered by linearizing the orthogonal dynamics equation. This allows for an analytic expression of the solution. The homogeneous portion of the linearized dynamics obeys

$$
\frac{d}{d t} \phi^{Q}=\mathbf{A}^{Q} \phi^{Q}
$$

where $\mathbf{A}^{Q}$ is a constant coefficient matrix specific to the linearized equations. Assuming $\mathbf{A}^{Q}$ to be diagonalizable, the linear system has the solution

$$
\phi^{Q}(t)=\mathbf{S} e^{\Lambda t} \mathbf{S}^{-1} \phi_{0}
$$


where $\Lambda$ and $\mathbf{S}$ are the eigenvalues and eigenvectors of $\mathbf{A}^{Q}$. The memory is then given by

$$
\int_{0}^{t} e^{(t-s) \mathcal{L}} \mathcal{P} \mathcal{L} e^{s Q \mathcal{L}} Q \mathcal{L} \phi_{0 j} d s=\int_{0}^{t} \mathcal{P} \mathcal{L} \mathcal{L} S_{j k} e^{\lambda_{k m} s} S_{m n}^{-1} \phi_{n}(t-s) d s .
$$

For cases where the eigenvectors and eigenvalues can be obtained analytically, or are independent of the initial conditions, the memory kernel can be evaluated.

Significant insight is gained from Eq. 15. It is seen that the convolution memory integrand contains the exponential term $e^{\lambda s}$. When the eigenvalues are negative (a general characteristic of stable systems), the exponential operator leads to the integrand having finite support. The timescale of this support is, in general, related to the argument of the exponential operator, which is related to the eigenvalues of the orthogonal dynamics equation.

\section{Approximating the Memory Kernel}

To obtain a reduction in complexity, a surrogate to the memory must be devised. The insight gained from Section III suggests that the memory integrand has a finite support. Based on this, a class of models that assume a finite support of the memory integrand is now considered. The models derived here are an extension of the models first discussed Stinis. ${ }^{13,14}$ For notational purposes, define

$$
w_{j}^{(0)}\left(\phi_{0}, t\right)=\mathcal{P} \int_{a_{0}(t)}^{t} e^{s \mathcal{L}} \mathcal{P} \mathcal{L} e^{(t-s) Q \mathcal{L}} Q \mathcal{L} \phi_{0 j} d s,
$$

where $a_{0}(t)=t-\tau_{0}(t)$. Note the change of variables $t^{\prime}=t-s$. For clarity of presentation, the dependence of $\hat{\phi}$ on the initial conditions $\phi_{0}$ and time $t$ will be implicitly assumed throughout the rest of this section. Differentiating Eq. 17 with respect to time yields

$$
\frac{d}{d t} w_{j}^{(0)}\left(\phi_{0}, t\right)=e^{t \mathcal{L}} \mathcal{P} \mathcal{L} Q \mathcal{L} \phi_{0 j}-e^{\left(t-\tau_{0}\right) \mathcal{L}} \mathcal{P} \mathcal{L} e^{\tau_{0} Q \mathcal{L}} Q \mathcal{L} \phi_{0 j} a_{0}^{\prime}(t)+\mathcal{P} \int_{a_{0}(t)}^{t} e^{s \mathcal{L}} \mathcal{P} \mathcal{L} e^{(t-s) Q \mathcal{L}} Q \mathcal{L} Q \mathcal{L} \phi_{0 j} d s .
$$

Note that the first term on the right hand side does not require the solution of the orthogonal dynamics equation. The second term on the right hand side is dependent on the orthogonal dynamics. This dependence can be eliminated by using a discrete integration scheme to express the memory integral. In the case of the trapezoidal rule

$$
w_{j}^{(0)}(t)=\left[e^{t \mathcal{L}} \mathcal{P} \mathcal{L} Q \mathcal{L} \phi_{0 j}+e^{\left(t-\tau_{0}\right) \mathcal{L}} \mathcal{P} \mathcal{L} e^{\tau_{0} Q \mathcal{L}} Q \mathcal{L} \phi_{0 j}\right] \frac{\tau_{0}(t)}{2}+O\left(\tau_{0}^{2}\right) .
$$

Applying the trapezoidal rule yields

$$
\frac{d}{d t} w_{j}^{(0)}\left(\phi_{0}, t\right)=-2 \frac{1-\tau_{0}^{\prime}}{\tau_{0}} w_{j}^{(0)}+\left(2-\tau_{0}^{\prime}\right) \mathcal{P} \mathcal{L} Q \mathcal{L} \phi_{j}(t)+\mathcal{P} \int_{t-\tau_{0}}^{t} e^{s \mathcal{L}} \mathcal{P} \mathcal{L} e^{(t-s) \mathcal{L} \mathcal{L}} Q \mathcal{L} Q \mathcal{L} \phi_{0 j} d s+O\left(\tau_{0}^{2}\right) .
$$

The right hand side of Eq. 19 is now closed with the exception of the new convolution integral. Assume that the integrand of the memory term has support $a_{1}(t) \leq a_{0}(t)$. Define

$$
w_{j}^{(1)}\left(\phi_{0}, t\right)=\mathcal{P} \int_{t-\tau_{1}}^{t} e^{s \mathcal{L}} \mathcal{P} \mathcal{L} e^{(t-s) \mathcal{Q} \mathcal{L}} Q \mathcal{L} Q \mathcal{L} \phi_{0 j} d s
$$

with $a_{1}(t)=t-\tau_{1}(t) \leq a_{0}(t)$. A differential equation for $w^{(1)}$ can be developed by again differentiating Eq. 20 with respect to time and using the trapezoidal rule. One obtains

$$
\begin{aligned}
\frac{d}{d t} w_{j}^{(1)}\left(\phi_{0}, t\right)=-2 \frac{1-\tau_{1}^{\prime}(t)}{\tau_{1}(t)} w_{j}^{(1)}(t)+\left[2-\tau_{1}^{\prime}(t)\right] \mathcal{P} \mathcal{L} Q \mathcal{L} Q \mathcal{L} \phi_{j}(t)+ \\
\qquad \mathcal{P} \int_{t-\tau_{1}}^{t} e^{s \mathcal{L}} \mathcal{P} \mathcal{L} e^{(t-s) \mathcal{L} \mathcal{L}} Q \mathcal{L} Q \mathcal{L} Q \mathcal{L} \phi_{0 j} d s+O\left(\tau_{1}^{2}\right) .
\end{aligned}
$$

This differentiation process can be continued to build an infinite hierarchy of Markovian equations that have the general form

$$
\frac{d}{d t} w_{j}^{(n)}\left(\phi_{0}, t\right)=-2 \frac{1-\tau_{n}^{\prime}(t)}{\tau_{n}(t)} w_{j}^{(n)}(t)+\left[2-\tau_{n}^{\prime}(t)\right] \mathcal{P} \mathcal{L}(Q \mathcal{L})^{n+1} \phi_{j}(t)+w_{j}^{(n+1)} .
$$


In practice, this infinite hierarchy of equations must be truncated at some point, which requires one to either model or ignore the effects of $w_{j}^{(n+1)}$. Simply neglecting the effect of $w_{j}^{(n+1)}$ may alarm the reader (as it should), but it can be justified if the support of the integrand in $w_{j}^{(n)}$ is acceptably small, or if the magnitude of $w_{j}^{(n)}$ is acceptably small. The derivation above can be carried out using multiple sub-intervals in the discrete integration, as well as with higher order discrete integration techniques. ${ }^{13}$

For completeness, the t-model is described. The t-model can be obtained by expanding the memory kernel in a Taylor series about $s=0$ and retaining only the leading term,

$$
\mathcal{P} \int_{0}^{t} e^{(t-s) \mathcal{L}} \mathcal{P} \mathcal{L} e^{s Q \mathcal{L}} Q \mathcal{L} \phi_{0 j} d s \approx \mathcal{P} \int_{0}^{t} e^{t \mathcal{L}} \mathcal{P} \mathcal{L} Q \mathcal{L} \phi_{0 j} d s=t \mathcal{P} \mathcal{L} Q \mathcal{L} \phi_{j}(t) .
$$

Despite its simplicity, the t-model has been successfully applied to Burgers equation and as a sub-grid model in Large Eddy Simulations. ${ }^{10}$

\section{Applications}

In this section, the methodology described previously is applied to to predictive simulations of the viscous Burgers equation and the incompressible Navier-Stokes equations. The expectation projection in the zero-variance limit is used for all projection operations.

\section{A. Viscous Burgers Equation}

The viscous Burgers equation (VBE) is a one-dimensional equation that serves as a simple analogue to the NavierStokes equations. The VBE in Fourier space is given by

$$
\frac{\partial u_{k}}{\partial t}+\frac{\imath k}{2} \sum_{\substack{p+q=k \\ p, q \in F \cup G}} u_{p} u_{q}=-v k^{2} u_{k}, \quad k \in F \cup G
$$

with $u_{k}(0)=u_{0 k}$. The Fourier modes $u=\{\hat{u}, \tilde{u}\}$ are written to belong to the union of two sets, $F$ and $G$. For the coarse-grained model, take the resolved modes $\hat{u} \in F$ and the unresolved modes $\tilde{u} \in G$. For the case of a sharp spectral cutoff, the filtered viscous Burgers equation can be found by partitioning Eq. 24 into the resolved and unresolved sets,

$$
\frac{\partial u_{k}}{\partial t}+\frac{\imath k}{2} \sum_{\substack{p+q=k \\ p \in F, q \in F}} u_{p} u_{q}=-v k^{2} u_{k}-\frac{\imath k}{2}\left(\sum_{\substack{p+q=k \\ p \in G, q \in G}} u_{p} u_{q}+\sum_{\substack{p+q=k \\ p \in F, q \in G}} u_{p} u_{q}+\sum_{\substack{p+q=k \\ p \in G, q \in F}} u_{p} u_{q}\right) \quad k \in F .
$$

Note that the sub-grid stress in Fourier space is written as

$$
\tau_{k}^{S G S}=\frac{1}{2}\left(\sum_{\substack{p+q=k \\ p \in G, q \in G}} u_{p} u_{q}+\sum_{\substack{p+q=k \\ p \in F, q \in G}} u_{p} u_{q}+\sum_{\substack{p+q=k \\ p \in G, q \in F}} u_{p} u_{q}\right) .
$$

The last term on the RHS of Eq. 25 represents the effect of the unresolved scales on the resolved scales and must be modeled.

Due to symmetries in the VBE, the high fidelity model is taken to have support $-N \leq k \leq N-1$, while the reduced order model has support for $-N / 2 \leq k \leq N / 2-1$. Evaluating Eq. 11 for the VBE yields

$$
\frac{\partial u_{k}}{\partial t}+\frac{\imath k}{2} \sum_{\substack{p+q=k \\ p \in F, q \in F}} u_{p} u_{q}=-v k^{2} u_{k}+\mathcal{P} \int_{0}^{t} e^{(t-s) \mathcal{L}} \mathcal{P} \mathcal{L} e^{s Q \mathcal{L}} Q \mathcal{L} u_{0 k} d s .
$$

The computational complexity of the problem is reduced by approximating the memory kernel. Results for the firstorder finite memory model and the t-model are presented. For the VBE the first order term is found to be

$$
e^{t \mathcal{L}} \mathcal{P} \mathcal{L} Q \mathcal{L} u_{0 k}=-\imath k \sum_{\substack{p+q=k \\ p \in F, q \in G}} u_{p}\left[-\frac{l q}{2} \sum_{\substack{r+s=q \\ r, s \in F}} u_{r} u_{s}\right] \quad k \in F .
$$


While the t-model requires no heuristics, the finite memory models require the specification of a memory length. Obtaining an a priori evaluation of the memory kernel is challenging for Burgers equation. Approximate analytic solutions for the orthogonal dynamics can be obtained through simplifications of the orthogonal dynamics equation, but here a simpler approach is used. It is known that the solution to the linearized orthogonal dynamics equation is given by a linear combination of the eigenvectors and eigenvalues. Since the Burgers equation does not exhibit scale separation, a simple hypothesis is to relate the time scale of the memory to the spectral radius of the Jacobian of the resolved variables,

$$
\tau \propto 1 / \rho\left(\frac{\partial \mathbf{R}}{\partial \mathbf{u}}\right) .
$$

A parametric study was performed to assess the validity of this hypothesis. In the study, the optimal time constant in the least squares sense was found by minimizing the difference of the total kinetic energy dissipation rate between coarse-grained solutions and a high resolution solutions for various simulation conditions. The study is discussed in the Appendix. The results of the study show that scaling the time-constant with the spectral radius of the the Jacobian of the resolved variables is a reasonable model, and results in the time constant being approximated as

$$
\tau_{0} \approx 0.2 / \rho\left(\frac{\partial \mathbf{R}_{0}}{\partial \mathbf{u}_{0}}\right)
$$

Note that the memory is taken to have a constant length.

Numerical simulations of the VBE were performed for various Reynolds numbers and resolutions. The simulations were performed on a spatially periodic domain of length $2 \pi$ for $t \in[0,2]$. The initial condition is given by ${ }^{15}$

$$
u(x)=U_{0}^{*} \sum_{i=1}^{k_{c}} \sqrt{2 E\left(k_{i}\right)} \sin \left(k_{i} x+\beta_{i}\right)
$$

where $E(k)=5^{-5 / 3}$ if $1 \leq k \leq 5$ and $E(k)=k^{-5 / 3}$ for $k>5$. Eq. 28 initializes an energy spectrum with a $-5 / 3$ slope for $k>5$. The phase angle $\beta$ is a random number in the domain $[-\pi, \pi]$. A constant seed value is used in all of the simulations. The summary of computational details and case parameters is given in Table 1.

\begin{tabular}{|l|l|l|l|l|l|l|}
\hline & $v$ & $U_{0}^{*}$ & $k_{c}$ & $\tau_{0}(\mathrm{FM} 1)$ & $N(\mathrm{DNS})$ & $N(\mathrm{LES})$ \\
\hline Case 1 & 0.01 & 1 & 16 & 0.135 & 2048 & 32 \\
\hline Case 2 & 0.01 & 5 & 8 & 0.087 & 2048 & 16 \\
\hline Case 3 & 0.0005 & 10 & 32 & 0.0084 & 4096 & 64 \\
\hline
\end{tabular}

Table 1: Summary of computational details for numerical simulations of Burgers equation.

The results of the numerical simulations are shown in Figures 1 and 2. The Mori-Zwanzig based models are compared to the Smagorinsky model (denoted by SS) and filtered DNS data, as well as additional simulations on the same LES mesh with no sub-grid model. The total kinetic energy in the resolved modes, dissipation of total kinetic energy, and the mean magnitude of the memory term are show in Figure 1. As expected, the simulations performed without any sub-grid model under-predict the rate of decay of kinetic energy as there is no mechanism to remove energy from the resolved scales. The t-model offers some improvement. The predictions for kinetic energy are significantly improved and the predicted memory term is accurate for early times. For late times, the t-model over predicts the content in the memory term, but qualitative similarities are still present. This late time error is likely due to the lack of damping provided by the memory kernel. The results of the finite memory models compare well with the DNS data for all three cases. The evolution of kinetic energy is well characterized, as is the memory prediction. The Mori-Zwanzig based models out-perform the Smagorinsky model for all cases.

A $k-t$ diagram of the spatio-temporal field of the memory spectrum for Case 3 is shown in Figure 2 . Both the t-model and the finite memory model are in good agreement with the DNS. In particular, the streaks seen in the $k-t$ diagram compare well with the DNS data. This feature is not seen in the Smagorinsky model. The t-model is seen to slightly over-predict the magnitude of the memory term at late times, as was evidenced in Figure 1 . The spatio-temporal field predicted by the finite memory model is close to that of the DNS. 

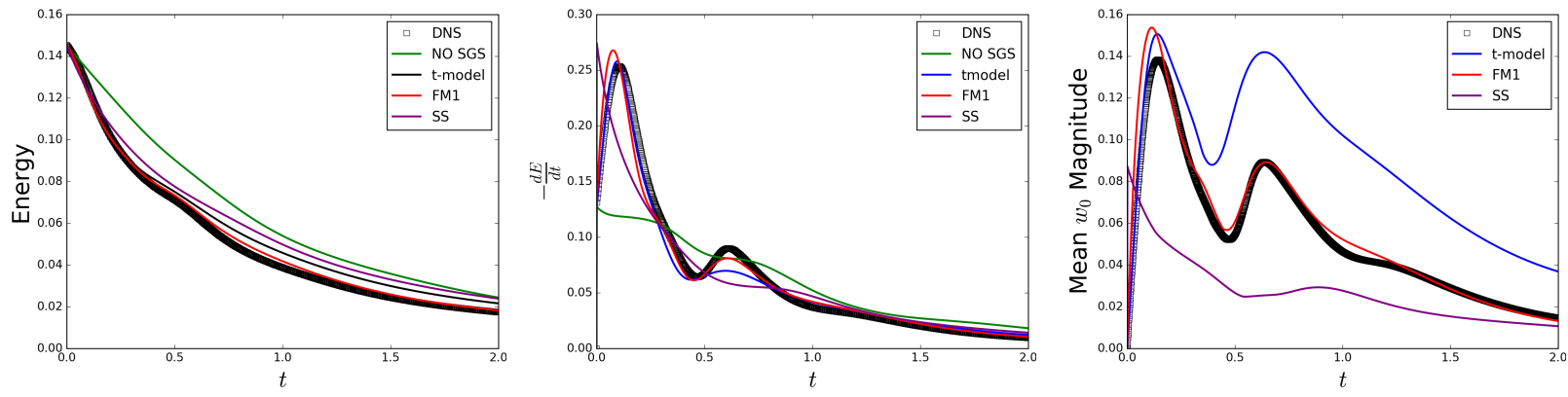

(a) Evolution of select quantities for $k_{c}=16, v=0.01, U_{0}^{*}=1$.
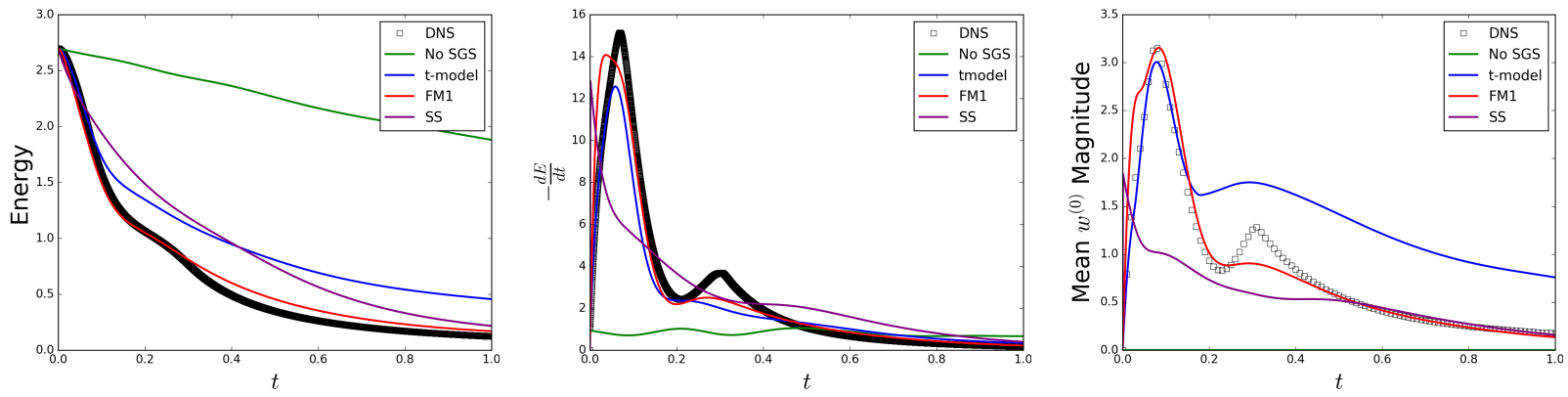

(b) Evolution of select quantities for $k_{c}=8, v=0.01, U_{0}^{*}=5$.
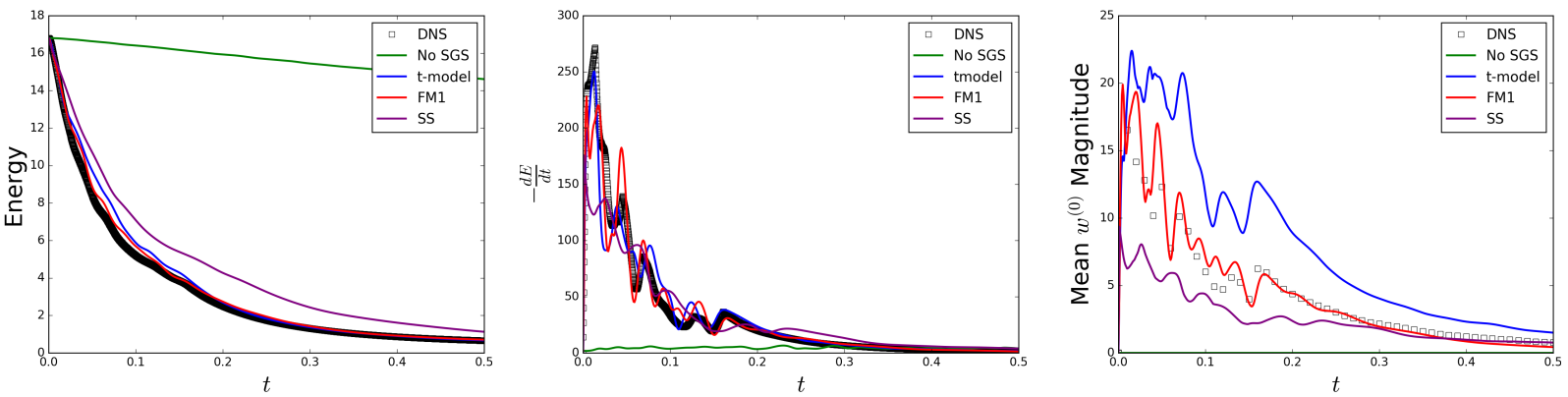

(c) Temporal evolution of total kinetic energy (left) and rate of decay of kinetic energy (right).

Figure 1: A comparison of Large Eddy Simulations performed with various sub-grid models to filtered DNS data. 


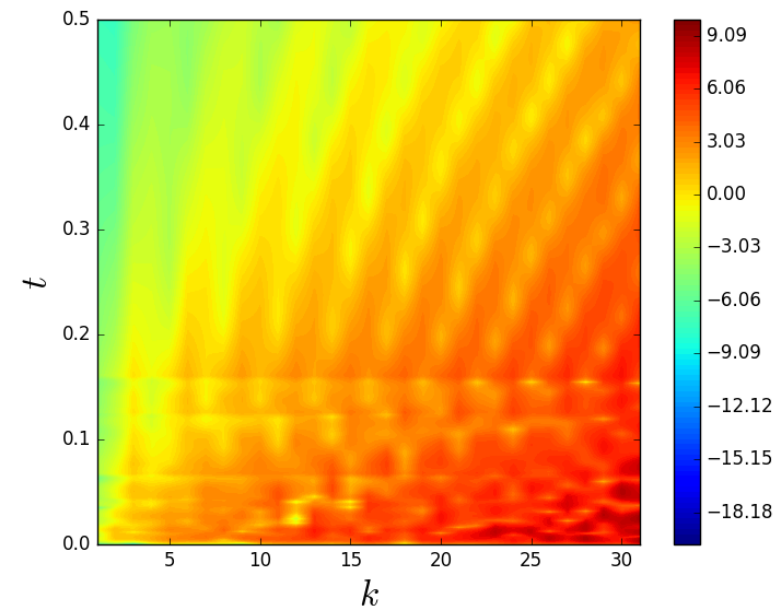

(a) DNS.

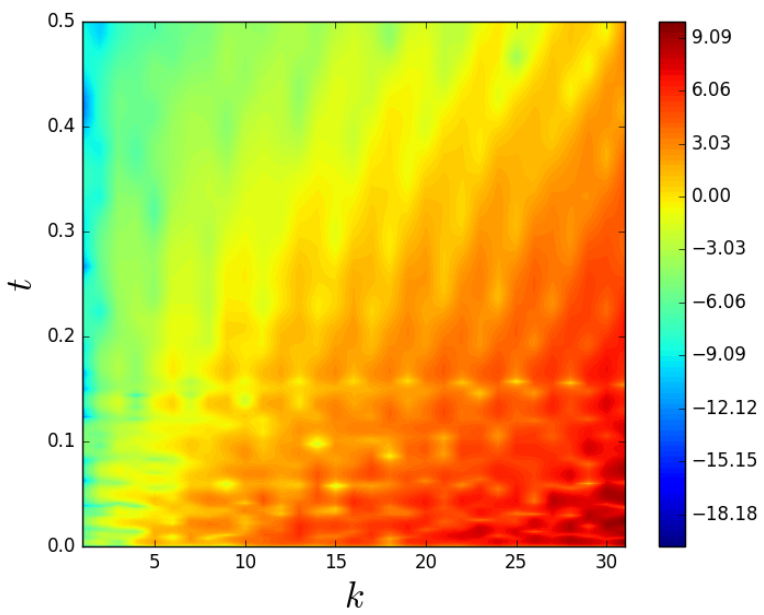

(c) Finite Memory.

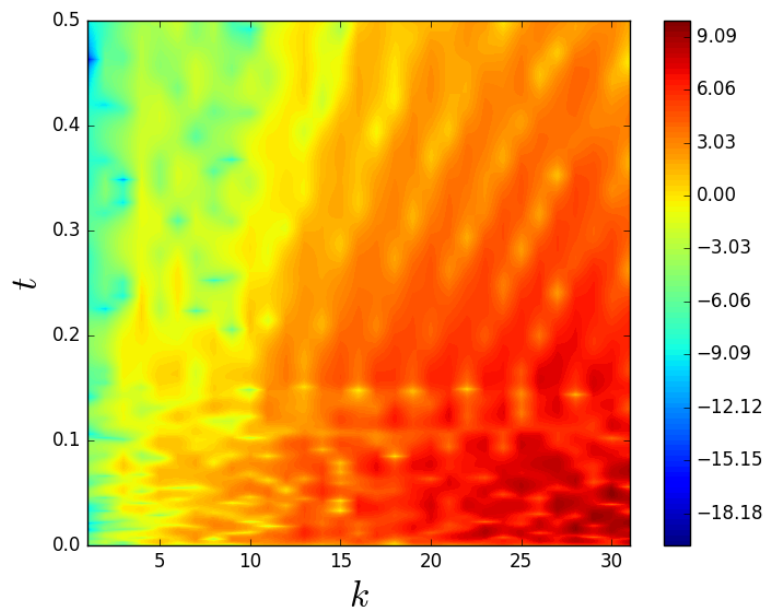

(b) t-model.

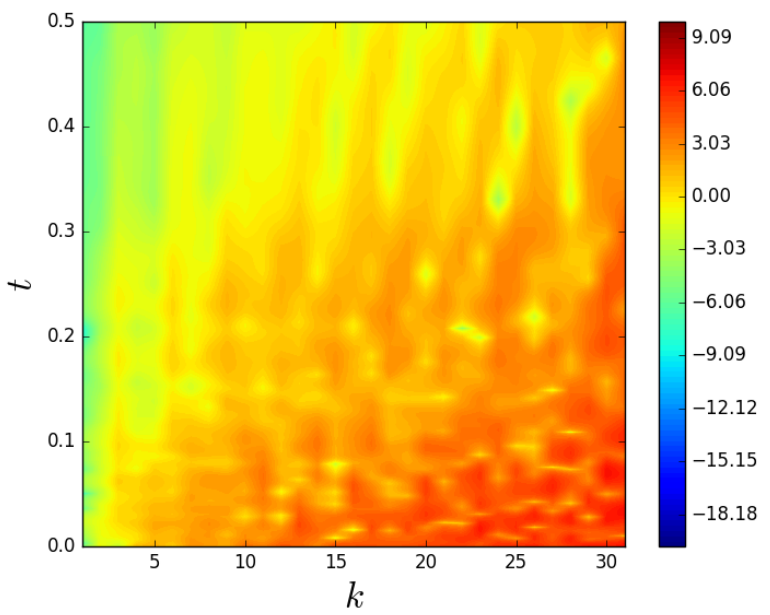

(d) Smagorinsky.

Figure 2: $k-t$ diagram of the memory spectrum, $\log \left(w^{(0)} w^{(0)^{*}}\right)$, as computed by various sub-grid models for Case 3 . 


\section{B. Incompressible Navier-Stokes Equations}

Fourier-Galerkin solutions of the Navier-Stokes equations are now considered. The incompressible Navier-Stokes equations in Fourier space are given by

$$
\left(\frac{\partial}{\partial t}+v k^{2}\right) \hat{u}_{i}(\mathbf{k}, t)+\left(\delta_{i m}-\frac{k_{i} k_{m}}{k^{2}}\right) \imath k_{j} \sum_{\substack{\mathbf{p}+\mathbf{q}=\mathbf{k} \\ \mathbf{p}, \mathbf{q} \in F \cup G}} \hat{u}_{j}(\mathbf{p}, t) \hat{u}_{m}(\mathbf{q}, t)=0 \quad \mathbf{k} \in F \cup G
$$

where the Fourier modes have again been written to belong to the union of resolved and unresolved sets. Separating the modes into these sets yields the filtered equations in the case of a sharp spectral cutoff filter,

$$
\left(\frac{\partial}{\partial t}+v k^{2}\right) \hat{u}_{i}(\mathbf{k}, t)+\left(\delta_{i m}-\frac{k_{i} k_{m}}{k^{2}}\right) \imath k_{j} \sum_{\substack{\mathbf{p}+\mathbf{q}=\mathbf{k} \\ \mathbf{p}, \mathbf{q} \in F}} \hat{u}_{j}(\mathbf{p}, t) \hat{u}_{m}(\mathbf{q}, t)=-\left(\delta_{i m}-\frac{k_{i} k_{m}}{k^{2}}\right) \imath k_{j} \tau_{j m}(\mathbf{k}, t) \quad k \in F,
$$

where $F$ are the resolved modes and $G$ are the unresolved modes. The sub-grid stress is written as

$$
\tau_{j m}(\mathbf{k}, t)=\sum_{\substack{\mathbf{p}+\mathbf{q}=\mathbf{k} \\ \mathbf{p}, \mathbf{q} \in G}} \hat{u}_{j}(\mathbf{p}, t) \hat{u}_{m}(\mathbf{q}, t)+\sum_{\substack{\mathbf{p}+\mathbf{q}=\mathbf{k} \\ \mathbf{p} \in G, \mathbf{q} \in F}} \hat{u}_{j}(\mathbf{p}, t) \hat{u}_{m}(\mathbf{q}, t)+\sum_{\substack{\mathbf{p}+\mathbf{q}=\mathbf{k} \\ \mathbf{p} \in F, \mathbf{q} \in G}} \hat{u}_{j}(\mathbf{p}, t) \hat{u}_{m}(\mathbf{q}, t) .
$$

Note that, in Fourier space, the pressure term appears as a projection. This projection leads to additional non-linear interactions between the resolved and unresolved scales.

Coarse-grained solutions using the t-model and first order finite memory model are considered. The expectation projection and Gaussian density in the zero variance limit are again used. The projected Mori-Zwanzig identity reads

$$
\left(\frac{\partial}{\partial t}+v k^{2}\right) \hat{u}_{i}(\mathbf{k}, t)+\left(\delta_{i m}-\frac{k_{i} k_{m}}{k^{2}}\right) i k_{j} \sum_{\substack{\mathbf{p}+\mathbf{q}=\mathbf{k} \\ \mathbf{p}, \mathbf{q} \in F}} \hat{u}_{j}(\mathbf{p}, t) \hat{u}_{m}(\mathbf{q}, t)=\mathcal{P} \int_{0}^{t} e^{s \mathcal{L}} \mathcal{P} \mathcal{L} e^{(t-s) Q \mathcal{L}} Q \mathcal{L} \hat{u}_{i} d s \quad k \in F .
$$

Both the t-model and the finite memory model require the evaluation of $\mathcal{P} \mathcal{L} Q \mathcal{L} u_{0 k}$. After much tedious algebra, it can be shown that

$$
\begin{aligned}
e^{t \mathcal{L}} \mathcal{P} \mathcal{L} \mathcal{L} \hat{u}_{i}(\mathbf{k}, 0)=\left(-\delta_{i m}+\frac{k_{i} k_{m}}{k^{2}}\right) \imath k_{j} \sum_{\substack{\mathbf{p}+\mathbf{q}=\mathbf{k} \\
\mathbf{p} \in F, \mathbf{q} \in G}} \hat{u}_{j}(\mathbf{p}, t) \mathcal{P} \mathcal{L} \hat{u}_{m}(\mathbf{q}, t)- & \left(\delta_{i m}+\frac{k_{i} k_{m}}{k^{2}}\right) \imath k_{j} \sum_{\substack{\mathbf{p}+\mathbf{q}=\mathbf{k} \\
\mathbf{p} \in F, \mathbf{q} \in G}} \hat{u}_{m}(\mathbf{p}, t) \mathcal{P} \mathcal{L} \hat{u}_{j}(\mathbf{q}, t),
\end{aligned}
$$

where

$$
\mathcal{P} \mathcal{L} \hat{u}_{i}(\mathbf{k}, t)=-\underbrace{\nu k^{2} \hat{u}_{i}}_{k \in G}(\mathbf{k}, t)-\left(\delta_{i m}-\frac{k_{i} k_{m}}{k^{2}}\right) \imath k_{j} \sum_{\substack{\mathbf{p}+\mathbf{q}=\mathbf{k} \\ \mathbf{p}, \mathbf{q} \in F}} \hat{u}_{j}(\mathbf{p}, t) \hat{u}_{m}(\mathbf{q}, t) .
$$

Eq. 32, along with Eqns. 23 and 18, can be used to write equations for the t-model and the first order finite memory model. It is noted that the terms $\mathcal{P} \mathcal{L} u_{i}$ are simply the right hand side of the filtered equations without any sub-grid model and are already computed. Additionally, when expanded, several terms in $\mathcal{P} \mathcal{L} Q \mathcal{L} u_{i}$ can be combined for a faster evaluation.

The spectral Navier-Stokes equations are solved using the pseudo-spectral method with an explicit low storage RK4 time integration scheme. All FFT calculations are de-aliased by the $3 / 2$ rule. For the Mori-Zwanzig models, a $2 x$ padding is used such that $F \in[-N / 2, N / 2-1]$ and $G \in([-N,-N / 2-1],[N / 2, N-1])$. Convolutions of the form

$$
\sum_{\substack{\mathbf{p}+\mathbf{q}=\mathbf{k} \\ \mathbf{p} \in F, \mathbf{q} \in G}} u_{j}(\mathbf{p}, t) u_{j}(\mathbf{q}, t),
$$

which have a support of $2 N$, are padded by construction. ${ }^{12}$

Simulations of Homogeneous Isotropic Turbulence are considered. The spectrum used by Rogallo ${ }^{16}$ is used for initialization. The initial velocity field is given by

$$
u_{i}(\mathbf{k})=\alpha e_{i}^{1}+\beta e_{i}^{2},
$$


where $e_{i}^{1}$ and $e_{i}^{2}$ are mutually orthogonal unit vectors in the plane orthogonal to the wave vector. The initial spectrum is taken to be

$$
E(k, 0)=\frac{q^{2}}{2 A} \frac{1}{k_{p}^{\sigma+1}} k^{\sigma} \exp \left(-\frac{\sigma}{2}\left(\frac{k}{k_{p}}\right)^{2}\right),
$$

where $k_{p}$ is the wave number at which the energy spectra is maximum, $\sigma$ is a parameter set to 4 , and $A=\int_{0}^{\infty} k^{\sigma} \exp \left(-\sigma k^{2} / 2\right) d k$.

Coarse-grained simulations are performed using $64^{3}$ resolved modes. The simulations are initialized with DNS data from a $512^{3}$ simulation that uses Eq. 33 as an initial condition. The M-Z models are compared to the dynamic and static Smagorinsky models, as well as a coarse-grained simulation using no sub-grid model. Computational details are given in Table 2.

\begin{tabular}{|l|l|l|l|l|}
\hline HIT & DNS & Smagorinsky & t-model & Finite Memory \\
\hline$N$ & $512^{3}$ & $64^{3}$ & $64^{3}$ & $64^{3}$ \\
\hline$\Delta t$ & 0.005 & 0.005 & 0.005 & 0.005 \\
\hline Constants & NA & $C s=0.16$ & NA & $\tau_{0}=0.1$ \\
\hline
\end{tabular}

Table 2: Summary of computational details for homogeneous isotropic turbulence case.

The results of the simulations are shown in Figure 3. The energy and dissipation spectra are shown in Figures 3a and $3 \mathrm{~b}$, respectively. The resolved and sub-grid contributions to the transfer spectra are shown in Figures $3 \mathrm{c}$ and $3 \mathrm{~d}$. The coarse-grained simulation not utilizing any sub-grid model has a pile-up of energy at high frequencies, a phenomenon that occurs due the lack of of mechanism to remove energy from the resolved modes. The Smagorinsky models both perform well, but the sub-grid contribution to the transfer-spectra is seen to be erroneous. The t-model performs well for low wave numbers, but error is present at high wave numbers. The transfer spectra has notable error for mid to high wave numbers, with too much energy being deposited into the mid wave numbers. The finite memory model again performs well. The energy and dissipation spectra predicted by the model are in good agreement with the DNS data. The sub-grid contribution to the energy transfer is notably better than the t-model or Smagorinsky models.

\section{Conclusions}

The Mori-Zwanzig (M-Z) formalism was introduced as a basis for sub-grid scale modeling for Large Eddy Simulations. While the M-Z approach provides a mathematically consistent framework it does not lead to a reduction in complexity as it contains unclosed terms. In this work, a methodology for solving the unclosed orthogonal dynamics equation was presented. Utilizing the insight gained from the solution of the orthogonal dynamics, a class of models based on the assumption that the memory convolution integral in the generalized Langevin equation has a finite, time dependent support was presented. These models are appealing in that they are derived from the governing equations themselves and require minimal heuristic arguments. The closure models were applied to the viscous Burgers equation and the incompressible Navier-Stokes equations.

The models were found to accurately approximate sub-grid content in the viscous Burgers equation and NavierStokes equations. The downside of the finite memory models is that they require the specification of a memory length. While the memory length has physical meaning and is more than heuristic tuning, the a priori selection of the memory length is challenging and requires insight into the orthogonal dynamics. Nonetheless, the performance of the models shows that there is merit to the Mori-Zwanzig approach. The models here should be considered to be a first order approximation. Further insight into the mechanics of the memory kernel may allow for a more complete surrogate representation of the integral. Additional complexities, such as spatio-temporal memory lengths and models for the unclosed term $w_{j}^{(m+1)}$, may improve the predictive capabilities of the models.

\section{Acknowledgments}

This research was supported by the National Science Foundation via grant 1507928. 


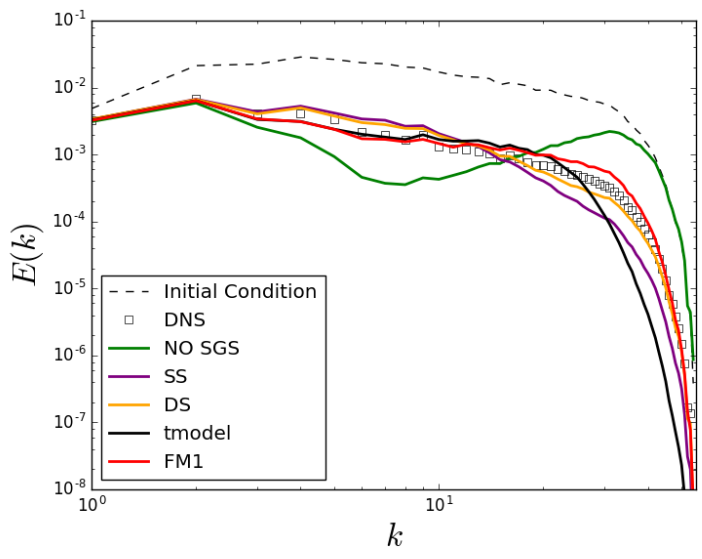

(a) Filtered energy spectra.

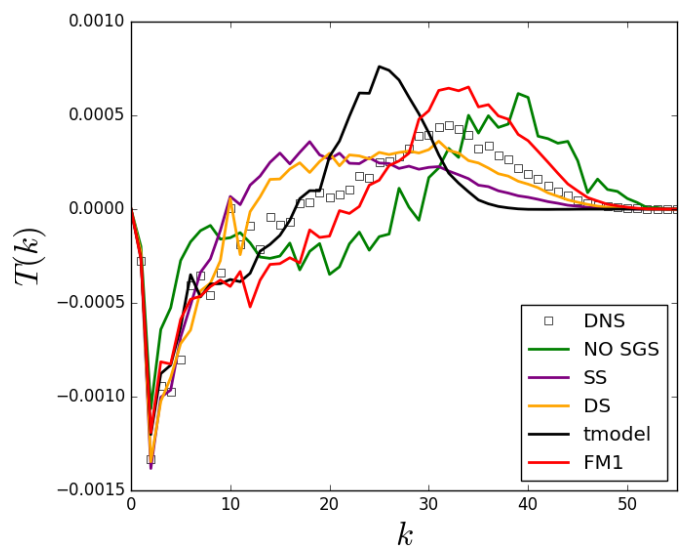

(c) Transfer spectra as computed by the resolved modes.

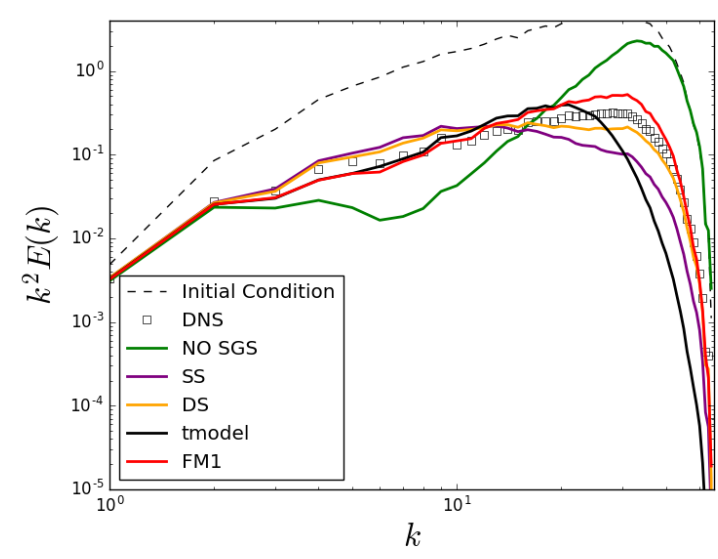

(b) Filtered dissipation spectra.

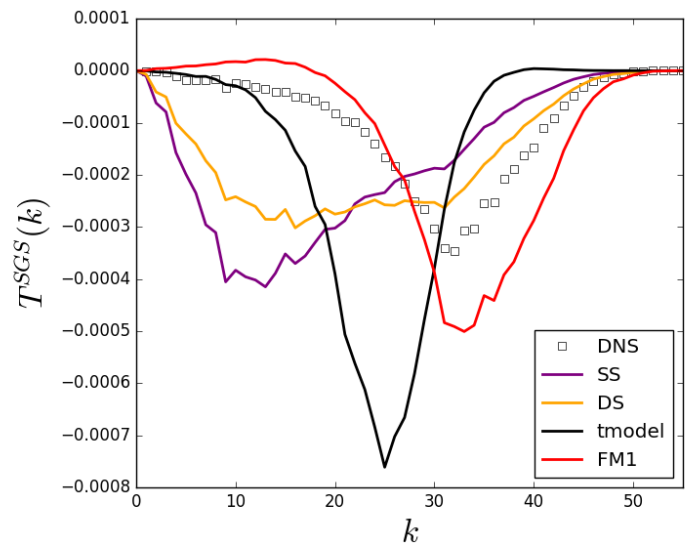

(d) Sub-grid contribution to the transfer spectra.

Figure 3: Energy, dissipation, and transfer spectra for homogeneous isotropic turbulence case. 


\section{Memory Length for Burgers Equation}

The finite memory models require the specification of a memory length. The insight gained by linearizing the orthogonal dynamics equation showed that the length of the memory is given by a linear combination of the eigenvalues of the orthogonal dynamics. For system that do not display scale separation, a logical hypothesis is that a mean time scale can be related to the spectral radius of the Jacobian of the resolved variables

$$
\tau \propto 1 / \rho\left(\frac{\partial \mathbf{R}}{\partial \mathbf{u}}\right) .
$$

The validity of this hypothesis was tested by performing a parametric study of Burgers equation involving a variety of simulations conditions. The simulations were initialized with Eq. 28 and operated over a range of Reynolds numbers and resolutions. The cases considered were permutations of the following parameters: $v=[0.05,0.01,0.005,0.001,0.0005], k_{c}=$ $[8,16,32], U_{0}^{*}=[1,2,5,10]$. The DNS simulations were carried out using 4096 resolved modes. For each case, the the optimal time constant in the least squares sense was found by minimizing the difference of the total kinetic energy dissipation rate between the reduced order model solution and a high resolution DNS solution. The solution was minimized for $t \in[0,2]$ using data at discrete time-steps spaced by intervals of $\Delta t=0.01$. The discrete penalty function is given by

$$
\mathcal{J}=\sum_{n=1}^{N}\left(\left[\frac{d K_{n}}{d t}\right]_{M-Z}-\left[\frac{d K_{n}}{d t}\right]_{D N S}\right)^{2}
$$

where $N=2 / 0.01=2000$. The penalty function was minimized using SciPy's optimization suite. A downhill simplex algorithm was used. The results of the inverse problem are summarized in Figure 4, which shows the inferred memory lengths $\tau_{0}$ plotted against the spectral radius of the Jacobian at time $t=0$ of the resolved variables. The data is seen to collapse to a linear line when $k_{c}$ is held constant. Slight variations are seen when $k_{c}$ is varied. Overall the collapse of the data shows that the Jacobian of the resolved scales can be used as an indicator of the memory length. A linear fit of the inferred data produces $\tau_{0} \approx 0.2 / \rho\left(\frac{\partial F}{\partial u}\right)$.

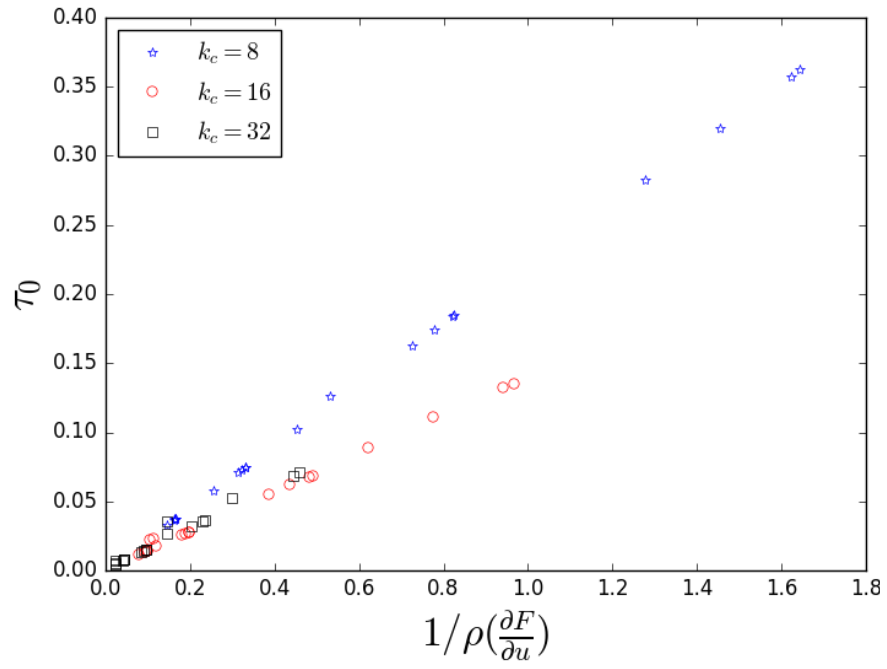

Figure 4: Inverse solution for the first order model time constant $\tau_{0}$ plotted against the inverse of the spectral radius of the Jacobian of the resolved variables.

\section{References}

\footnotetext{
${ }^{1}$ Zwanzig, R., "Nonlinear generalized Langevin equations," Journal of Statistical Physics, 1973, pp. 215-220.

${ }^{2}$ Mori, H., "Transport, collective motion, and Brownian motion," Prog. Theoret. Phys, 1965, pp. 423-250.

${ }^{3}$ Chorin, A. and Stinis, P., "Problem reduction, renormalization, and memory," Commun. Appl. Math. Comput. Sci., 2006, pp. $239-257$.

${ }^{4}$ Chorin, A., Hald, O., and Kupferman, R., "Optimal prediction with memory," Phys. D, 2002, pp. 239-257.

${ }^{5}$ Chorin, A. J. and Hald, O. H., "Stochastic Tools for Mathematics and Science," 2009.
} 
${ }^{6}$ Chorin, A. J., Hald, O., and Kupferman, R., "Optimal prediction and the Mori-Zwanzig representation of irreversible processes," Proc. Natl Acad. Sci., Vol. 97, No. (doi:10.1073/pnas.97.7.2968), 2000, pp. 2968-2973.

${ }^{7}$ Barber, J. L., Application of Optimal Prediction to Molecular Dynamics, Ph.D. thesis, University of California, Berkeley, CA, 2004.

${ }^{8}$ Givon, D., Hald, O. H., and Kupferman, R., "Existence Proof for Orthogonal Dynamics and the Mori-Zwanzig Formalism," Israel Journal of Mathematics, 2005, pp. 221-241.

${ }^{9}$ Bernstein, D., "Optimal Prediction of Burgers's Equation," Multiscale Model. Simul., Vol. 6, No. 1, 2007, pp. $27-52$.

${ }^{10}$ Chandy, A. J. and Frankel, S. H., "The t-model as a Large Eddy Simulation model for the Navier-Stokes Equations," Multiscale Model. Simul., Vol. 8, No. 2, 2009, pp. 445-462.

${ }^{11}$ Stinis, P., "Renormalized Mori-Zwanzig-reduce models for systems without scale separation," Proceedings of The Royal Society, 2015.

${ }^{12}$ Stinis, P., "Higher order Mori-Zwanzig models for the Euler equations," arXiv:math/0607108v1, 2006.

${ }^{13}$ Stinis, P., "Mori-Zwanzig reduced models for uncertainty quantification I: Parametric uncertainty," arXiv preprint, Vol. 1211.4285, 2012.

${ }^{14}$ Hald, O. H. and Stinis, P., "Optimal prediction and the rate of decay for solutions of the Euler equations in two and three dimensions," Proceedings of the National Academy of Sciences, 2007.

${ }^{15} \mathrm{Li}$, Y. and Wang, Z., "A Priori and A Posteriori Evaluations of Subgrid Stress Models with the Burgers' Equation," 53rd AIAA Aerospace Sciences Meeting, Kissimmee, Florid, 5-9 January 2015.

${ }^{16}$ Rogallo, R. S., "Numerical Experiments in Homogeneous Turbulence," Technical Memorandum 81315, NASA, September 1981. 